\title{
Three-dimensional modeling of beam emission spectroscopy measurements in fusion plasmas
}

\author{
D. Guszejnov, ${ }^{1, \text { a) }}$ G. I. Pokol, ${ }^{1, \text { b) }}$ I. Pusztai, ${ }^{2}$ D. Refy, ${ }^{3}$ S. Zoletnik, ${ }^{3}$ M. Lampert, ${ }^{1,3}$ and Y. U. Nam ${ }^{4}$ \\ 1) Department of Nuclear Techniques, Budapest University of Technology and Economics, Association EURATOM, \\ H-1111 Budapest, Hungary \\ ${ }^{2)}$ Nuclear Engineering, Applied Physics, Chalmers University of Technology, SE-412 96 Göteborg, \\ Sweden \\ ${ }^{3)}$ MTA Wigner FK RMI, Association EURATOM, Pf. 49, H-1525 Budapest, \\ Hungary \\ 4) National Fusion Research Institute, Gwahangno 113, Daejeon 305-333, Republic of Korea
}

(Dated: 1 February 2018)

One of the main diagnostic tools for measuring electron density profiles and the characteristics of long wavelength turbulent wave structures in fusion plasmas is Beam Emission Spectroscopy (BES). The increasing number of BES systems necessitated an accurate and comprehensive simulation of BES diagnostics, which in turn motivated the development of the RENATE simulation code that is the topic of this paper. RENATE is a modular, fully three-dimensional code incorporating all key features of BES systems from the atomic physics to the observation, including an advanced modeling of the optics. Thus RENATE can be used both in the interpretation of measured signals and the development of new BES systems. The most important components of the code have been successfully benchmarked against other simulation codes. The primary results have been validated against experimental data from the KSTAR tokamak.

Keywords: Beam emission spectroscopy, simulation, rate equations, RENATE, density measurements, fluctuation measurements, fusion plasmas, diagnostics

\section{INTRODUCTION}

Although fusion has been an intensely explored area for over 50 years, still several key phenomena, such as the turbulent transport, spontaneous transition to enhanced confinement regimes or the edge localized modes, have not been adequately explained. Also a solid predictive capability regarding to the confinement properties of fusion plasmas is still lacking. The progress in the development and testing of theoretical models and predictive simulation codes strongly relies on measurements from various plasma diagnostics. In particular there is a need for accurate, well localized measurements of density profiles and fluctuations, which can be accomplished by Beam Emission Spectroscopy (BES). BES is an active plasma diagnostic which analyzes the collisionally induced emission of either a non-intrusive Diagnostic Neutral Beam (DNB) probe or in some cases that of a Neural Beam Injection (NBI) heating beam ${ }^{1}$. The emission distribution provides information about the distribution of density in the plasma.

Having good spatial resolution (few cms) and high sampling rate (few $\mathrm{MHz})^{1}$ BES is ideal for measuring density fluctuations making it the basic diagnostic tool for studying the long wavelength fluctuations of the plasma turbulence and different transient transport events. This resulted in a high demand for such systems. Since determining the viability of a BES mea-

\footnotetext{
a)Electronic mail: guszejnov@reak.bme.hu

b) Electronic mail: pokol@reak.bme.hu
}

surement configuration is not trivial, several simulation packages have been in use to aid such efforts. The emitted light intensity can be directly calculated from beam and plasma parameters if the cross sections of relevant atomic physics processes are provided. A number of codes using collisional-radiative models ${ }^{2,3}$ have been developed which can provide the local emission intensity, such as FLYCHK ${ }^{4}, \mathrm{NOMAD}^{5}$, $\mathrm{SIMULA}^{6}, \mathrm{NUBEAM}^{7}$, $\mathrm{ALCBEAM}^{8}$ and the code developed by O. Marchuk ${ }^{9}$. However these are atomic physics simulation packages and accordingly the effects of the observation and beam geometry are not taken into account. From these aspects, a more detailed approach is taken in Simulation of Spectra ${ }^{10}$, which uses a less precise quasi-steady approximation $^{3}$, but accounts for spectral effects (e.g. motional Stark-effect) and includes basic observation and device geometry.

The development of new BES systems and the evaluation of current measurements required a detailed simulation which takes all geometrical effects, including observation, beam structure and plasma parameter distributions into account, allowing it to directly simulate measured signals. This motivated the development of the RENATE simulation code. The aim of the current paper is to give an overview of RENATE by providing information about its capabilities and potential applications.

The remainder of the paper is organized as follows. In Section II we describe the main structure of the code along with the theoretical models used. Then, in Section III an example will be given for the application of RENATE along with the validation of RENATE results against a particular experimental setup on KSTAR. Finally, the results are discussed in Sec. IV. 


\section{THE RENATE SIMULATION CODE}

In the present section we give an overview of the BES simulation code, RENATE (Rate Equations for Neutral Alkali-beam Technique). The code has a modular structure and is written in the IDL language ${ }^{11}$. As the name suggests, the original purpose of RENATE was the modeling of alkali atomic beams (lithium and sodium) ${ }^{12}$, but during the course of its development support for the more common hydrogen isotopes was also implemented. RENATE has two different atomic physics kernels, both allowing the modeling of the beam evolution in plasmas with mixed isotope content and arbitrary impurity composition.

RENATE is capable of calculating beam evolution by using a collisional-radiative model ${ }^{2,3}$, taking collisional excitation (exc), de-excitation (dexc), ionization (ion), charge exchange $(C X)$ reactions and spontaneous deexcitation into account. The recombination of the ionized beam material and the interaction with the background electromagnetic radiation field are neglected. This leads to the following time dependent rate equations in the frame moving with the beam atoms:

$$
\begin{gathered}
\frac{d N_{i}}{d t}=\sum_{I} n_{I}\left[-N_{i}\left(\sum_{j=i+1}^{m} R_{I}^{e x c}(i \rightarrow j)+\sum_{j=1}^{i-1} R_{I}^{\text {dexc }}(i \rightarrow j)+R_{I}^{i o n}(i)+R_{I}^{C X}(i)\right)+\right. \\
\left.\left(\sum_{j=1}^{i-1} N_{j} R_{I}^{e x c}(j \rightarrow i)+\sum_{j=i+1}^{m} N_{j} R_{I}^{\text {dexc }}(j \rightarrow i)\right)\right]-N_{i} \sum_{j=1}^{i-1} A(i \rightarrow j)+\sum_{j=i+1}^{m} N_{j} A(j \rightarrow i),
\end{gathered}
$$

where $N_{i}$ is the population of the atomic level $i$ ( $i=1$ denotes the ground state and $m$ is the number of considered levels), $n_{I}$ is the density of species $I, R$ denotes the rate coefficients and $A$ denotes the Einstein coefficients. The rate coefficients are calculated from the cross sections $(\sigma)$ by assuming that the plasma species have a Maxwellian population while the beam is assumed to be monoenergetic.

$$
R=\langle\sigma v\rangle=\int f(T, \boldsymbol{v})\left|\boldsymbol{v}-\boldsymbol{v}_{b}\right| \sigma\left(\left|\boldsymbol{v}-\boldsymbol{v}_{b}\right|\right) d \boldsymbol{v},
$$

where $\boldsymbol{v}_{b}$ is the beam velocity, $\boldsymbol{v}$ is the speed of the particle species interacting with the beam, while $f(T, \boldsymbol{v})$ is their Maxwellian velocity distribution. Equation (1) is solved by assuming the plasma parameters to be constant during the time needed for the beam to pass the observed region. This allows the transformation of Eq. (1) into a space dependent system of differential equations, which can be written in the following matrix form:

$$
\frac{d \mathbf{N}}{d x}=\left[\mathbf{A}(x) n_{e}(x)+\mathbf{B}\right] \cdot \mathbf{N}(x)=\mathbf{C}(x) \cdot \mathbf{N}(x),
$$

where the $\mathbf{B}$ matrix contains the spontaneous atomic transitions while the $\mathbf{A}(x)$ matrix contains all collisional processes. $\mathbf{A}$ is dependent on the spatial location $x$ through the temperature and impurity distribution. Due to the aforementioned transformation rule the simulation of gas jets is not feasible using Eq. (3) as the prescribed minimum beam velocity is too high. Eq. (3) is solved by RENATE using a fourth order Runge-Kutta scheme.

In case of hydrogen isotope beams the cross sections for $(n, l) \rightarrow(n, l \pm 1)$ transitions are so high, that a statistical population between the sub-shells can be assumed, this is called the bundled-n approximation ${ }^{13}$ that we adopt (here $n$ and $l$ denote the principal and azimuthal quantum numbers, respectively). Due to the fact that only a finite number of atomic levels can be taken into account, RENATE solves Eq. (1) by considering only the first 9 levels in case of lithium, 8 levels in case of sodium and 6 levels (shells) in case of hydrogenic species. This approximation is based on the fact that higher levels tend to be only lightly populated (see Fig. 1) due to the small cross sections and high rate of spontaneous de-excitement. $\mathrm{Nu}$ merical calculations have shown that increasing the number of considered levels above the number considered by RENATE only negligibly affects the emission distribution (e.g. $<1 \%$ in case of hydrogen beams).

The rate coefficients are calculated by the atomic physics kernel of RENATE, using atomic physics data from several sources. The cross sections for lithium were taken from Schweinzer et al. ${ }^{14}$, while data for sodium were obtained from Igenbergs et al. ${ }^{15}$. Cross section data for hydrogenic species were obtained from the IAEA ALADDIN $^{16}$ and the Open ADAS $^{17}$ databases with the corrections from E. Delabie and O. Marchuk ${ }^{18}$.

It should be noted that, unlike in the quasi-stationary model to be discussed later, the rate coefficients for the impurity ions are scaled from the rates of hydrogenic plasma species. Both theoretical calculations and experiments show that the cross section of various processes between impurities and plasma species exhibit universal behavior which is only dependent on the charge $(q)$ and energy $(E)$ of the ion ${ }^{14,19}$. The scaling stipulates that the reduced cross section $\left(\sigma_{p}^{r e d}\right)$ of process $p$ at the reduced energy $\left(E^{r e d}\right)$ is identical for all species. The reduced cross sections and energies for excitation, charge 
exchange and ionization processes are the following

$$
\begin{array}{cc}
\sigma_{e x c}^{r e d}=\frac{\sigma_{e x c}}{q} & E_{\text {exc }}^{r e d}=\frac{E_{e x c}}{q}, \\
\sigma_{C X}^{r e d}=\frac{\sigma_{C X}}{n_{e f f}^{4} q} & E_{C X}^{r e d}=\frac{n_{e f f}^{2} E_{C X}}{q}, \\
\sigma_{i o n}^{r e d}=\frac{\sigma_{i o n}}{n_{e f f}^{4} q^{1.3}} & E_{i o n}^{r e d}=\frac{n_{\text {eff }}^{2} E_{i o n}}{\sqrt{q}},
\end{array}
$$

where $n_{\text {eff }}=\sqrt{R y / E_{b}}$ is the effective principal quantum number with $R y$ as the Rydberg constant and $E_{b}$ as the binding energy.

The rate equation solver of RENATE has been benchmarked against the code developed by O. Marchuk ${ }^{9}$ for hydrogenic beams, and against the SIMULA code ${ }^{6}$ for lithium beams (Fig. 1), yielding identical results within the numerical accuracy of the calculations.

Instead of solving the rate equations RENATE can also utilize a simpler quasi-stationary model ${ }^{3}$, taking atomic physics data from the Open ADAS database ${ }^{17}$. This model, that has low computational requirements, provides a quick and approximate solution to the problem of the beam evolution. It assumes the life-time of excited atomic levels to be negligible compared to the time scale of beam evolution. The beam attenuation according to the quasi-stationary model is given by

$$
\begin{aligned}
N(t) & =\sum_{i=1}^{\infty} N_{i}(t)=N_{0} \cdot e^{\int_{t_{0}}^{t} S_{\text {eff }}(\tau) d \tau} \\
S_{\mathrm{eff}}(\tau) & =\sum_{a} n_{a}(\tau) S_{a}\left(n_{a}(\tau), T_{a}(\tau), E\right),
\end{aligned}
$$

where $N(t)$ is the total number of non-ionized beam particles, $N_{i}(t)$ is the population of the atomic level $i$, and $S_{a}\left(n_{a}, T_{a}, E\right)$ denotes the beam stopping coefficient corresponding to the interaction between the beam and plasma species $a$ calculated from rate coefficients ${ }^{3}$

$$
S_{a}\left(n_{a}, T_{a}, E\right)=1 /\left[\mathbf{C}_{a}^{-1}\left(n_{a}, T_{a}, E\right)\right]_{1,1},
$$

using the notation of Eq. (3).

The emission at a given point is taken to be proportional to the number of excited particles in the relevant atomic state and the local density, that is

$$
\lambda_{n \rightarrow n^{\prime}}(\mathbf{r})=\epsilon_{n \rightarrow n^{\prime}}\left(n_{e}(\mathbf{r}), T_{e}(\mathbf{r}), E\right) \cdot n_{e}(\mathbf{r}) \cdot N(\mathbf{r}),
$$

where $\lambda_{n \rightarrow n^{\prime}}(\mathbf{r})$ is the emission density corresponding to the $n \rightarrow n^{\prime}$ transition at a specific spatial position while $\epsilon_{n \rightarrow n^{\prime}}\left(n_{e}, T_{e}, E\right)$ is the effective emission coefficient.

$$
\epsilon_{n \rightarrow n^{\prime}}\left(n_{e}, T_{e}, E\right)=A_{n \rightarrow n^{\prime}}\left(N_{n}^{(S)} / N_{e} N_{1}\right) F_{n}^{(1)},
$$

where $A_{n \rightarrow n^{\prime}}$ is the Einstein coefficient, $N_{n}^{(S)}$ is the SahaBoltzmann population for principal quantum shell $n$ and $F_{n}^{(1)}$ is the effective contribution of ground level excitations to the population of level $n^{3}$.
Both the beam stopping and effective emission coefficients are provided by the OpenADAS atomic physics database ${ }^{17}$. Since the quasi-stationary model neglects the finite life-time of excited atomic levels, the results are posteriorly smoothed by assuming exponential decay for the excited population to account for the effect. In practice this means that the emission distribution along the beamline is convolved with an exponential decay whose characteristic length is set to be the same as the observed excited state's.

The basic assumption of the quasi-static model is that the atomic relaxation is much faster than the rate of plasma parameter change which is proportional to the beam velocity. In some cases this can be violated in the plasma pedestal leading to erroneous results (Fig. 2).

While there are quite a few simulation codes which can calculate beam evolution ${ }^{4,5,10}$, some even in $3 \mathrm{D}^{7,8}$, RENATE is also able to take all the subtle geometrical effects of the observation into account. RENATE calculates the beam evolution along the beamline in three dimensions, while assuming the plasma density, temperature and impurity composition to be flux functions and distributing them according to the provided profiles and magnetic geometry of the configuration.

As it was previously mentioned, BES diagnostic measurements are performed not only on thin diagnostic beams but on heating beams as well. The latter - unlike probe beams - are in general not localized to a poloidal plane, hence the need for a full 3D simulation. Heating beams require large and complex ion sources, thus the 3D structure of the neutral beam is not necessarily trivial (as shown in Fig. 3), which in turn could have a significant effect on the measured signal. To account for this, the neutral beam itself is modeled as a set of infinitesimally thin virtual beams, for which the beam evolution is calculated individually in the 3D model of the tokamak.

Having calculated the emission distribution along each virtual beam, contributions to the observation channels are summed up by the optical modules. There are two possible choices for the observation modeling, representing different levels of sophistication. The simpler module utilizes a pinhole optics approximation which assumes that each channel of the optical system can only detect light that was emitted within a pyramid whose apex is the observation point, see Fig. 4. The amount of light reaching the detector surface is assumed to be equivalent to that reaching the forward aperture, which is calculated by integrating the emission density weighted by the geometrical efficiency factor (coming from aperture distance, size and orientation) within the observed volume of space.

The second, more sophisticated optical module uses ray-tracing through the Zemax ${ }^{20}$ model of the optical system to calculate the observation efficiency for every point in space, thus the transfer matrix of the optical system (Fig. 5). This involves a Monte Carlo simulation - carried out by Zemax - that creates a large number of randomly generated rays originating from a point-like source. These are traced through the optical system, tak- 

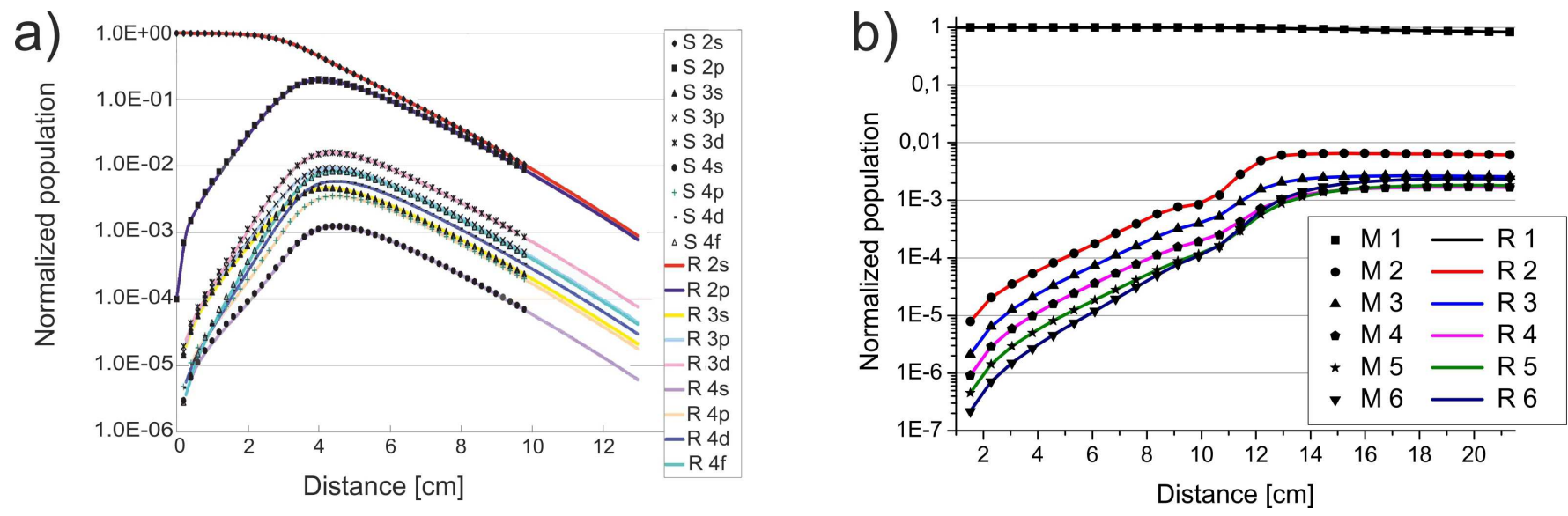

FIG. 1: Evolution of the population of atomic levels along the beam normalized to the initial particle number. Figure (a) shows the evolution of a lithium beam calculated by RENATE (R) and Simula (S). Figure (b) shows the evolution of a hydrogen beam calculated by RENATE $(\mathrm{R})$ and the code of O. Marchuk (M).

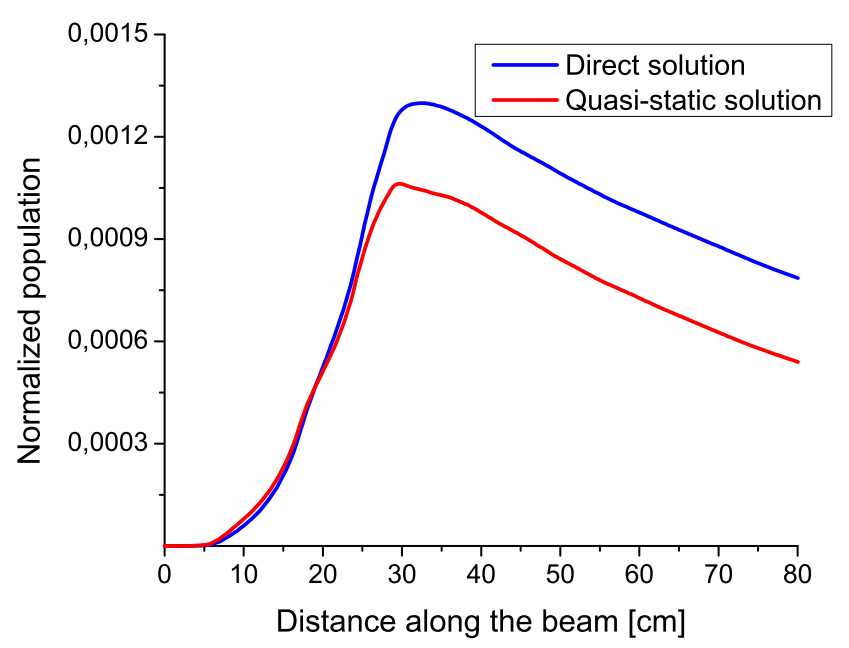

FIG. 2: Evolution of the population of the observed atomic level along the beam normalized to the initial particle number for KSTAR configuration detailed in Section III.

ing the characteristics of each optical element into account. Then the observation efficiency is simply the light intensity reaching the individual detector surface divided by the emitted intensity.

This method provides more accurate results, as it takes all the optical elements of the system into account, however it can only be used in the final design phase of BES systems when the detailed optical plan is available. Meanwhile the pinhole optics module is much more flexible allowing the tryout of a multitude of different configurations without the computationally demanding calculation of transfer matrices. It should be noted that while RENATE can calculate the Doppler-shifted spectrum and thus take filtering into account, it is currently unable to take other wavelength shifting effects (e.g. motional

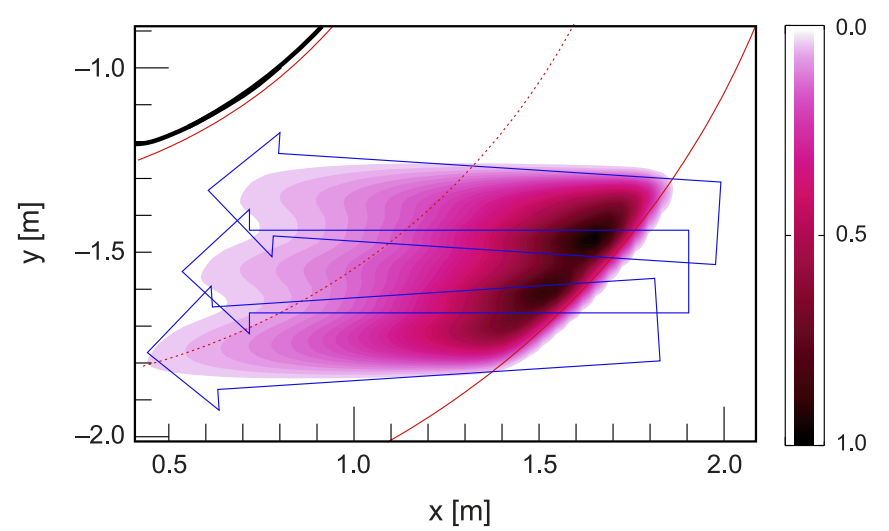

FIG. 3: Toroidal projection of the simulated relative emission intensity (purple tone color coding) of a heating beam with three ion sources (directions with blue arrows) in a hypothetical KSTAR configuration, also depicting magnetic axis (dotted red) and the edge of the plasma (red).

Stark-effect) into account, which means that filtering efficiency for each channel has to be provided externally (e.g. by another simulation code, like Simulation of Spectra ${ }^{10}$ ).

The final result of the calculation is the expected absolute photon currents $(1 / \mathrm{s})$ reaching the surface of the individual detector segments (Fig. 6).

To give a complete account of the capabilities of RENATE, we note that it can compute the density perturbation response matrices, which in turn could be used to determine the response in detected signal of the investigated BES system to arbitrary density perturbations. Using this matrix, the plasma density fluctuations can be converted into signal fluctuations, however this is only possible if the response is proportional to the strength of the perturbation. It is also possible to try and calculate the density fluctuations for a given BES system from the 


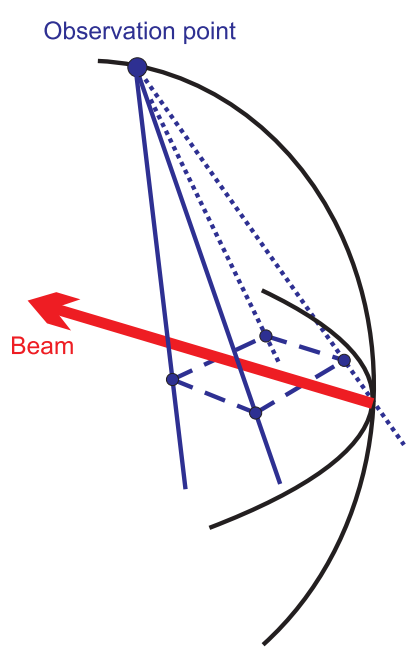

FIG. 4: Schematic figure of the pinhole optical model of RENATE showing the pyramid shaped observed area of space, the beamline and the observation point.

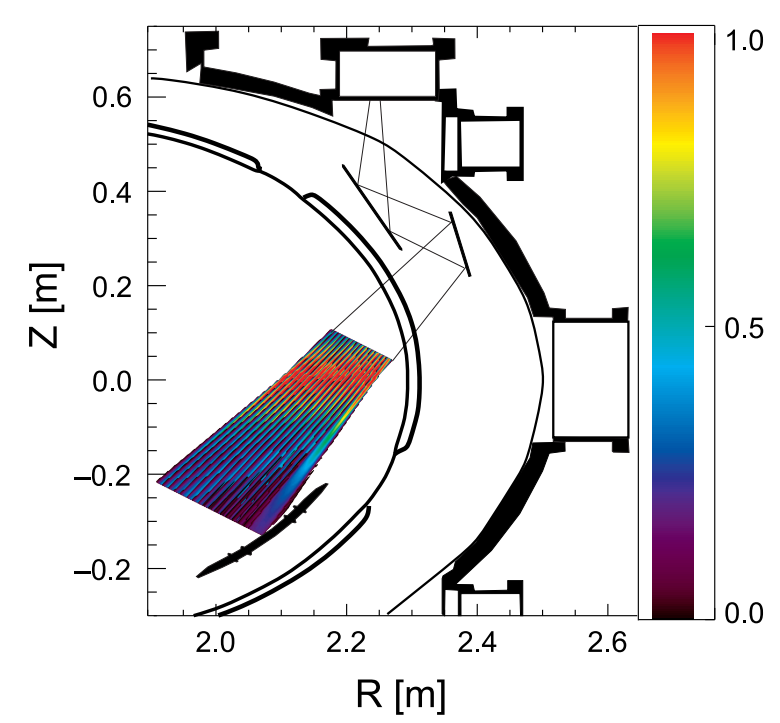

FIG. 5: Contour plot of the poloidal projection of the transfer matrices of each detector channel created by

the advanced optical module of RENATE in a

hypothetical TEXTOR configuration, also denoting the structural elements of the device and the optical paths of the terminal line of sights.

experimental signal data. This aspect of the RENATE simulation code will be thoroughly explored in a follow up article.

\section{APPLICATION TO KSTAR TRIAL MEASUREMENT}

The first demonstration of the capabilities of RENATE includes applications to the KSTAR BES system ${ }^{21}$. With the help of RENATE the photon currents reaching the de-

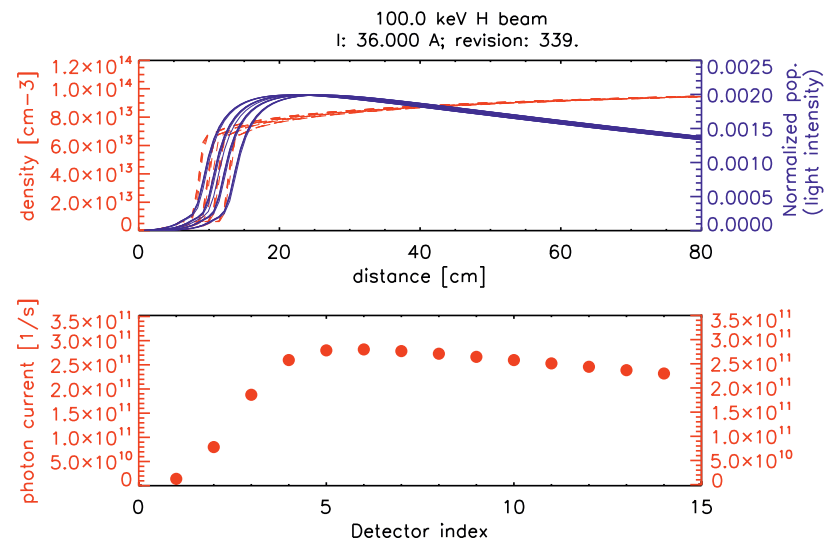

FIG. 6: Graphical output of RENATE showing the density along the beamlets (top-red), light profiles

(top-blue) and the photon currents reaching the detector surfaces (bottom)

tector surfaces could be calculated, which - combined with the given detector type's characteristics - enabled the calculation of the signal-to-noise ratio for each detector segment. RENATE was also capable of providing estimates of the $3 \mathrm{D}$ spatial and $2 \mathrm{D}$ poloidal resolution of the fluctuation BES system, by calculating the the response matrix and integrating it along magnetic field lines. The poloidal and toroidal projections of the simulated configuration are depicted on Fig. 7.

Using measured data the simulations have been rerun for the parameters of KSTAR discharge \#6123 at $1744 \mathrm{~ms}$, and its results have been compared against experimental results from the KSTAR BES trial measurement ${ }^{22}$. The magnetic geometry was reconstructed using EFIT $^{23}$ while the temperature profile was obtained from Electron Cyclotron Emission (ECE) measurements, thus assuming $T_{i}=T_{e}$ (Fig. 8). Since at the time of the measurements KSTAR had only a single interferometry channel for plasma density measurement, it was not possible to get localized density data from the experiment. Instead a profile of specific shape was fitted to the line integrated data. This density profile is flat in the core plasma, while follows the shape of the relative BES emission in the edge as shown in Fig. 8. Impurity data were also unavailable thus a homogeneous $1 \%$ carbon impurity was assumed, corresponding to $Z_{\text {eff }}=1.35$.

During the experiment only the central beam source was active, resulting in a single deuterium beam (13 A, $90 \mathrm{keV}$ ) with known current distribution. The measurement was carried out using a 4 × 8 matrix of detectors with spatial resolution of $1 \mathrm{~cm}$. Subtracting the background light from the measured data and taking into account the transmittance of the optical system along with the effect of the filtering and detector amplification yielded the absolute photon current reaching the aperture for each individual channels. This method however, carries a significant relative error mainly because only a 

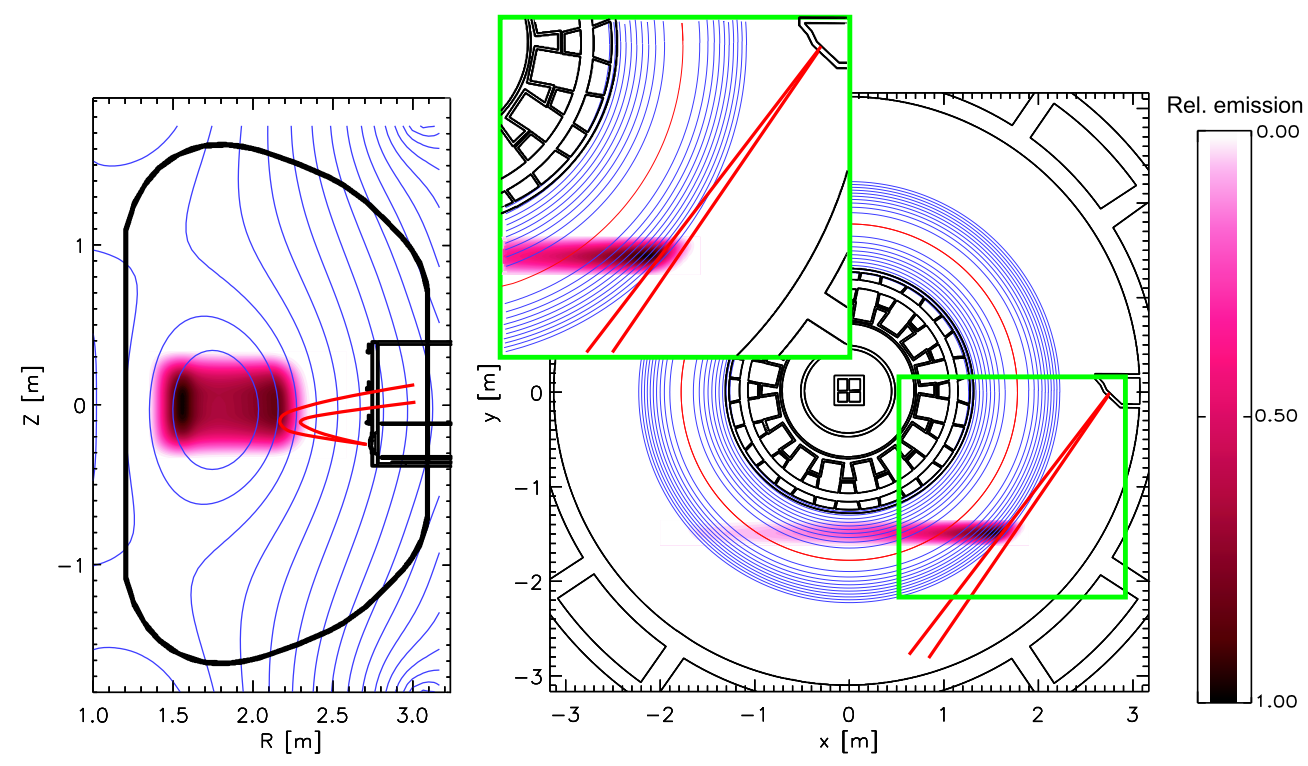

FIG. 7: Toroidal and poloidal projections of the simulated configuration for KSTAR BES, showing some structural elements (black), the flux surfaces (blue), the magnetic axis (red circle), the relative emission density (purple tone color coding) and the edge lines of sights (red) from the observation point

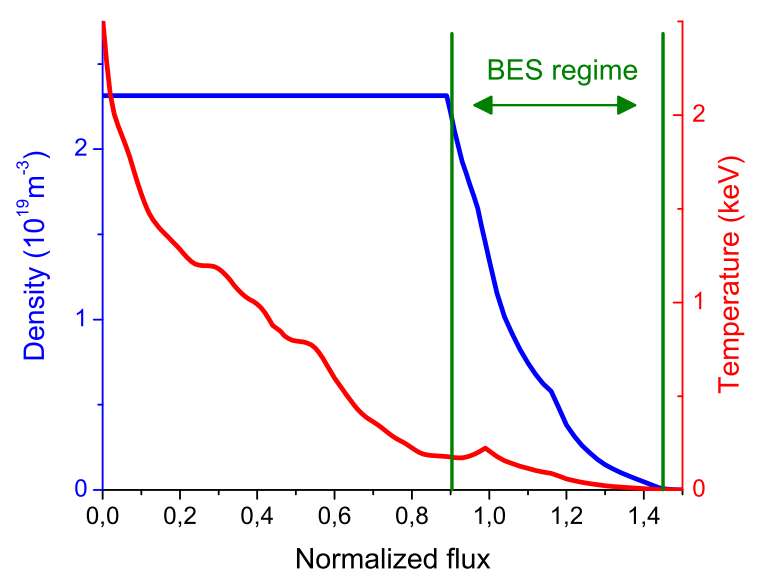

FIG. 8: Plasma parameter profiles used in the BES modeling of the KSTAR BES trial measurement. Density data (blue) were fitted to interferometry results while temperature data (red) were obtained from ECE measurements.

relative calibration of the detector amplification was possible from gas shots. By estimating this error term to be roughly $30 \%$ and assuming the other terms to be independent, the measured data have a total $33 \%$ relative error. These data can be compared against RENATE results as shown in Fig. 9. The density profile of this simulation was highly arbitrary and a basic pinhole optics module

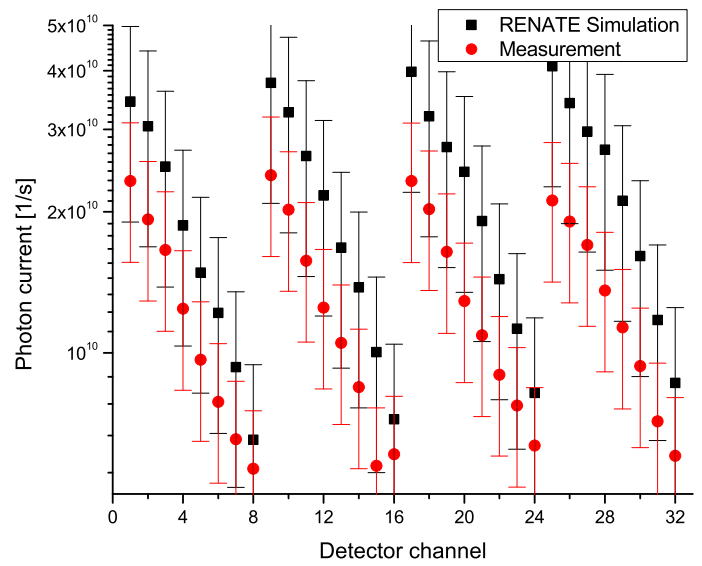

FIG. 9: Measured and simulated photon currents for individual detector channels for KSTAR \#6123 at 1744 ms. The detectors are sorted into a 4 x 8 matrix, indexing start from the detector of the first row observing the innermost part of the plasma. The error estimates are high due to inaccurate input data and experimental uncertainties.

was used to model the observation, which represent error terms of $40 \%$ and $20 \%$ respectively. This means an estimated relative error of $44 \%$ for the the simulation results.

As shown in Fig. 9 the simulated signal is close to the measurement. The upper part of Fig. 10 shows the 


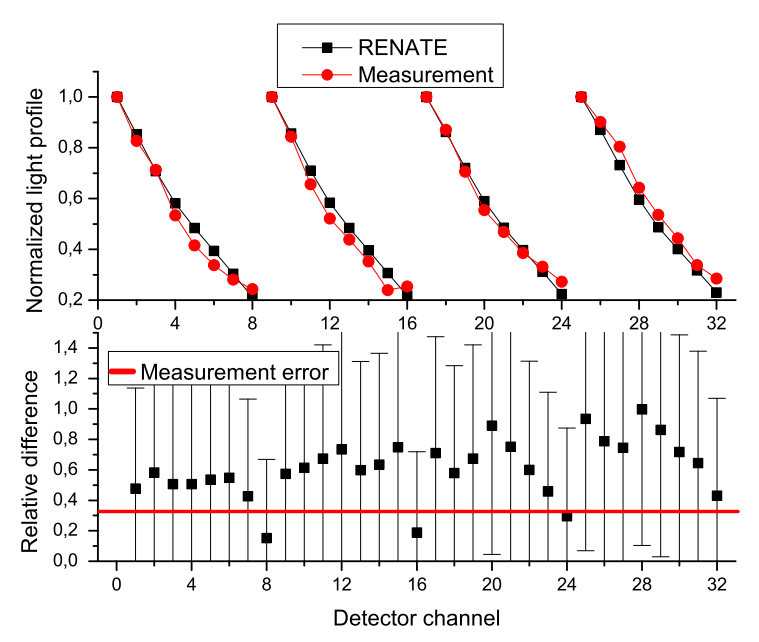

FIG. 10: Normalized light profiles of the individual detector rows (upper figure) and the relative error of the simulation compared to the measurement (lower figure).

normalized light profiles of the individual detector rows, where it is evident that the shape of the simulated signal matches that of the measurement. This is no surprise as the density profile was fitted to the relative BES emission which is roughly proportional to the local density as shown by Equation (3). The real test of the code is the lower part of Fig. 10, which shows the relative error of the simulation compared to the measurement.

On average the simulated results are $60 \%$ higher than the measurement. Considering that the standard deviation of the error is rather small (20\%) the dominant term is caused by a systematic error which can be caused by a number of effects.

- The density profile is the main source of error as it is the primary input of RENATE simulations. Since only line integrated density was available, the shape of the density profile in the non-BES region of the plasma could cause a systematic error. Using a parabolic profile for the plasma center instead of the flat one shown in Fig. 8 could mitigate this effect. It was found that a $40 \%$ reduction of the BES region density eliminates this term leaving only an error of only $20 \%$, which could easily be attributed to other uncertainties (e.g. pinhole optics model).

- The RENATE simulation utilized a simple pinhole optical model which could have easily neglected a portion of the real observed area.

- The atomic physics data of most hydrogenic transitions have a relative error of $10-20 \%^{16,17}$.

\section{CONCLUSIONS}

This paper presents an overview of the RENATE code, which is designed to help the development of new BES systems and the evaluation of experimental data. It is a modular BES simulation capable of simulating most key levels of a BES system. It possesses two different types of atomic physics kernel, allowing it to calculate beam evolution either by solving the rate equations or by employing a quasi-steady approximation. Both of these methods utilize the latest atomic physics data. RENATE is able to model the three-dimensional geometry of the simulated system in detail, including the inner structure of the beam and the optical setup, allowing it to model complete BES systems, in particular heating beam measurements.

The rate equation solver module of RENATE has been benchmarked for hydrogenic species and lithium. Meanwhile the experimental results for the KSTAR BES trial measurement agree with the simulated results well within the estimated error. In the future RENATE is to be compared against other experimental results and is to be upgraded to be able to take more wavelength shifting effects (e.g. motional Stark-effect) into account.

\section{ACKNOWLEDGMENTS}

This work was partly funded by the European Communities under Association Contract between EURATOM and HAS. The views and opinions expressed herein do not necessarily reflect those of the European Commission. Authors of this paper also acknowledge the support of the Hungarian National Development Agency under Contract No. NAP-1-2005-0013. We are grateful for the support of E. Delabie, O. Marchuk and J. Schweinzer.

${ }^{1}$ D. M. Thomas, G. R. McKee, K. H. Burrell, F. Levinton, E. L. Foley, and R. K. Fisher. Fusion Science and Technology, 53(2):488 - 527, 2008.

${ }^{2}$ B. Scwheer. Fusion Science and Technology, 53:425-432, 2008.

${ }^{3}$ H. Anderson, M. G. von Hellermann, R. Hoekstra, L. D. Horton, A. C. Howman, R. W. T. Konig, R. Martin, R. E. Olson, and H. P. Summers. Plasma Physics and Controlled Fusion, 42(7):781, 2009.

${ }^{4}$ H.-K. Chung, M.H. Chen, W.L. Morgan, Y. Ralchenko, and R.W. Lee. High Energy Density Physics, 1(1):3 - 12, 2005.

${ }^{5}$ Yuri V. Ralchenko and Yitzhak Maron. Journal of Quantitative Spectroscopy and Radiative Transfer, 71:609 - 621, 2001.

${ }^{6} \mathrm{~J}$. Schweinzer. Documentation on the Installation of a Code Package at NIFS for Reconstructing Density Profiles from Lithium Beam Emission Data. MPI für Plasmaphysik, Garching, 2005.

${ }^{7}$ Alexei Pankin, Douglas McCune, Robert Andre, Glenn Bateman, and Arnold Kritz. Computer Physics Communications, 159(3):157 - 184, 2004.

${ }^{8}$ I.O. Bespamyatnov, W.L. Rowan, and K.T. Liao. Computer Physics Communications, 183(3):669 - 676, 2012.

${ }^{9}$ O. Marchuk, G. Bertschinger, W. Biel, E. Delabie, M. G. von Hellermann, R. Jaspers, and D. Reiter. Review of Scientific Instruments, 79:10F532, 2008.

${ }^{10}$ M.G. von Hellermann, R. Jaspers, W. Biel, O. Neubauer, N. Hawkes, Y. Kaschuck, V. Serov, S. Tugarinov, D. Thomas, 
and W. Vliegenthart. In Proceedings of the 21st IAEA Conference, Chengdu, 16-21 October 2006, volume IAEA-CN-149 of IAEA Conference and Symposium Papers, pages IT/P1-26. IAEA, Vienna, 2007.

${ }^{11}$ ITT Data Visualization Solutions. IDL official webpage. http://www.ittvis.com/ProductServices/IDL.aspx, May 2010.

${ }^{12}$ I. Pusztai, G. Pokol, D. Dunai, D. Réfy, G. Pór, G. Anda, S. Zoletnik, and J. Schweinzer. Review of Scientific Instruments, 80(8):083502, 2009.

${ }^{13}$ A. Burgess and H. P. Summers. Monthly Notices of the Royal Astronomical Society, 174:345 - 391, 1976.

${ }^{14}$ J. Schweinzer, R. Brandenburg, I. Bray, R. Hoekstra, F. Aumayr, R.K. Janev, and HP. Winter. Atomic Data and Nuclear Data Tables, 72(2):239 - 273, 1999.

${ }^{15}$ K. Igenbergs, J. Schweinzer, I. Bray, D. Bridi, and F. Aumayr. Atomic Data and Nuclear Data Tables, 94(6):981 - 1014, 2008.

${ }^{16}$ IAEA. ALADDIN. http://www-amdis.iaea.org/ALADDIN/,
May 2010.

${ }^{17}$ ADAS Project. Open ADAS. http://open.adas.ac.uk, January 2011.

${ }^{18}$ E Delabie, M Brix, C Giroud, R J E Jaspers, O Marchuk, M G O'Mullane, Yu Ralchenko, E Surrey, M G von Hellermann, K D Zastrow, and JET-EFDA Contributors. Plasma Physics and Controlled Fusion, 52(12):125008, 2010.

${ }^{19}$ H. Ryufuku, K. Sasaki, and T. Watanabe. Phys. Rev. A, 21(3):745-750, Mar 1980.

${ }^{20}$ Warren J. Smith. Modern Optical Engineering. McGraw-Hill., 4 edition, 2007.

${ }^{21}$ D. Guszejnov and G. I. Pokol. First Results of RENATE Simulation for the KSTAR Tokamak. In Joint Workshop for KSTAR BES Diagnostics (Budapest), 2011.

${ }^{22}$ Y. U. Nam, S. Zoletnik, M. Lampert, and Á. Kovácsik. Review of Scientific Instruments, 83(10):10D531, 2012.

${ }^{23}$ L.L. Lao, J.R. Ferron, R.J. Groebner, W. Howl, H. St. John, E.J. Strait, and T.S. Taylor. Nuclear Fusion, 30(6):1035, 1990. 